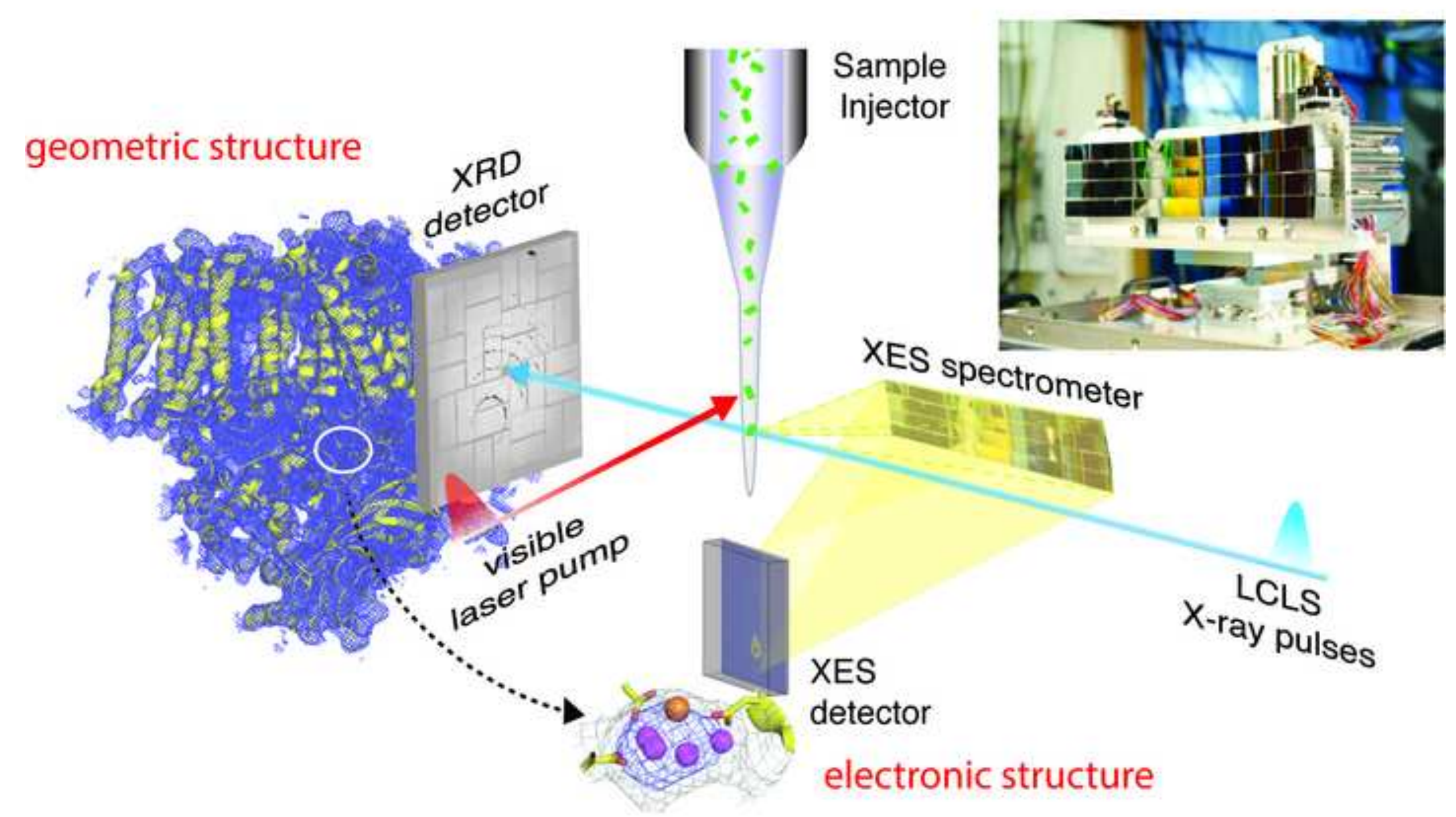

geometric structure electronic structure

detector

.




\title{
Metalloprotein structures at ambient conditions and in real-time: biological crystallography and spectroscopy using X-ray free electron lasers
}

\author{
Jan Kern ${ }^{1,2}$, Vittal K. Yachandra ${ }^{1}$, Junko Yano ${ }^{1}$
}

\section{Addresses:}

${ }^{1}$ Physical Biosciences Division, Lawrence Berkeley National Laboratory, Berkeley, CA 94720, USA.

${ }^{2}$ Linac Coherent Light Source, SLAC National Accelerator Laboratory, Menlo Park, CA, 94025, USA.

\section{Corresponding Authors:}

Jan Kern (JFKern@LBL.GOV), Vittal K. Yachandra (VKYachandra@LBL.GOV), Junko Yano (JYano@LBL.GOV) 


\begin{abstract}
Although the structure of enzymes and the chemistry at the catalytic sites have been studied intensively, an understanding of the atomic-scale chemistry requires a new approach beyond steady state X-ray crystallography and X-ray spectroscopy at cryogenic temperatures. Following the dynamic changes in the geometric and electronic structure of metallo-enzymes at ambient conditions, while overcoming the severe X-ray damage to the redox active catalytic center, is key for deriving reaction mechanisms. Such studies become possible by the intense and ultra-short femtosecond (fs) X-ray pulses from an X-ray free electron by acquiring a signal before the sample is destroyed. This review describes the recent and pioneering uses of XFELs to study the protein structure and dynamics of metallo-enzymes using crystallography, scattering, as well as the chemical structure and dynamics of the catalytic complexes (charge, spin, and covalency) using spectroscopy during the reaction to understand the electron-transfer processes and elucidate the mechanism.
\end{abstract}




\section{Introduction}

Metalloenzymes are key to many metabolic processes in all living organisms, including respiration, photosynthesis and nitrogen fixation. By incorporating one or several metal ions these enzymes significantly extend the chemistry that can be catalyzed, and over the course of evolution the bioinorganic catalysts at the active sites have been perfected to catalyze chemically demanding transformations under ambient conditions and with minimum driving force. Using Xrays to study these catalytic, and often, redox-active systems allows for the elucidation of the geometric structure but in addition also can provide information about the electronic structure of the active site, by using element selective X-ray spectroscopy methods. Nevertheless, the interaction with X-rays modifies the sample under study over the course of X-ray exposure, and therefore, the information is generally always that of a X-ray modified structure or a mixture of the native and modified states [1-3]. This "damage" occurs by absorption of X-ray photons by the atoms in the sample, leading to the generation of ionized species and radicals, with the absorption being dependent both on the energy of the X-ray photons (wavelength) and on the element. The spread of radiation damage can occur due to different mechanisms, the most prominent being the generation of free radicals in the aqueous buffer that then can migrate and create a cascade of redox/damage processes and it was found that the amount of radiation damage observed is predominantly dependent on the dose and not on the irradiation time (or dose rate). This situation changed with the recent advent of novel $4^{\text {th }}$ generation X-ray sources, the X-ray free electron lasers (XFEL) [4,5]. Here the possibility arose to probe such labile systems with high X-ray doses in an ultra-fast fashion extracting the information before manifestation of the X-ray induced changes to the sample, i.e. outrunning the slower damage processes by ultrafast data collection. This approach, originally termed "diffract before destroy" for diffraction experiments [6], can be generalized into "measure before modify" although novel damage mechanisms, different from the diffusion of radicals encountered during measurements at synchrotron sources have to be taken into account. These include the generation of plasma (due to the high dose rates and doses used), the generation of highly ionized species and the 
resulting Coulomb explosion (see section "Establishing structures of undamaged metal sites") [6,7].

$\mathrm{X}$-ray free electron lasers produce high intensity X-ray pulses in the fs time regime. This is achieved by the bunching of highly accelerated electrons in a long straight undulator section, leading to micro bunching. The interaction between these electron bunches and the X-rays emitted initially from the same bunch lead to a stimulated emission process, generating highly coherent and ultra short bursts of X-rays [8]. In practice the number of photons in a single $<50 \mathrm{fs}$ pulse is comparable to the number of photons available per second in a modern $3^{\text {rd }}$ generation synchrotron end station.

\section{Sample delivery methods}

In order to use the "measure before modify" method of data collection, it is necessary to expose a fresh volume of sample for each X-ray pulse. This field has rapidly evolved over the last five years, and several different approaches have been developed to achieve this sample replacement and they are tailored for different needs (e.g. X-ray pulse repetition rate, sample environment conditions). The traditional approach is the use of a goniometer-based setup similar to those that are used on protein crystallography beam lines at synchrotron sources. In this method, a large sample mount (either containing one large crystal or a grid of smaller crystals) is mounted on a goniometer and then rastered through the beam (Figure 1). In this approach it is for example possible to measure multiple spots from one larger crystal at known orientations with respect to each other [9-11]. The disadvantage is the slower repetition rate $(0.5$ to $10 \mathrm{~Hz})$, but on the other hand slower response detectors with a higher dynamic range can be used. So far all reported applications using this method have been performed using crystals frozen in a cryostream at $100 \mathrm{~K}$ but it should be easily possible to extend this approach to room temperature measurements, provided one can avoid sample dehydration and movement of the crystals in the grid during the measurement Similar to the goniometer method is the use of solid targets for

measurements in vacuum environments. Here the samples are placed onto small X-ray 
transparent windows on a larger target and then sealed against the vacuum using either a second window or e.g. vacuum grease or oil and this approach was used for initial studies of $2 \mathrm{D}$ and $3 \mathrm{D}$ crystals [12-14].

The most commonly used approach is a liquid jet (Figure 2). Here the jet diameter can be adapted to the X-ray beam diameter and sample size and this approach can be used to measure data from small samples, e.g. micro to nano crystals without a large background contribution from the surrounding buffer and mount. In common for all jets is the challenge to spatially overlap the micron sized jet with the micron sized X-ray beam and to actually obtain a reasonable high hit rate of samples. Due to the pulsed nature and low repetition rate of the X-ray source and the continuous flow of the jet X-rays probe only a very small fraction of the jet volume, leading to sample waste. Even with high particle densities in the jet the probability of hitting a single particle in each shot is low leading to a large number of empty detector images and typical hit rates are in the few percent range. There are different types of jets used, including gas-focusing jets [15,16], electro-focusing jets [17,18] and lipidic cubic phase jets $[19,20]$, each of them having different advantages and disadvantages. Whereas gas-focusing jets are in general more easily to establish and run very stable they often require a higher flow rate and some buffer compositions, for example high salt concentrations could lead to failures due to salt deposition and drying at the injector nozzle. In contrast electro-focusing jets offer lower flow rates but it is often necessary to add chemicals (e.g. glycerol) to the sample solution in order to increase the viscosity and prevent drying/freezing of the jet. Lipidic cubic phase jets and similar high viscosity jets have the advantage of very low flow rates but the high viscosity medium has to be compatible with the sample, and the jets show a higher scattering background and are to slow for measurements at higher X-ray repetition rates, when reformation of the jet does not occur between two X-ray pulses. All of the above mentioned sample delivery methods can be used for crystalline as well as for solution samples, although some of the jet methods have clear restrictions on the maximum size of the suspended particles that can be used and in general the upper bound for crystals measured using jet methods so far is $\sim 20 \mu \mathrm{m}$. For samples that are 
available in large volumes, it is also possible to use classical Raleigh jets, where a large sample diameter can be achieved, but at the price of a high flow rate $(\mathrm{ml} / \mathrm{min})$, compared to $\mu 1 / \mathrm{min}$ rates possible with the jets described earlier. In addition aerosol injectors have been used for single particle imaging experiments with XFEL pulses, where it was possible to generate a stream of individual particles (e.g. viruses or whole cells) intersecting the X-ray path [21-24].

\section{Establishing fs X-ray crystallography}

The first study to establish protein crystallography with fs X-ray pulses was performed at the LCLS by measuring shot-by-shot diffraction from a stream of $0.2-2 \mu \mathrm{m}$ sized crystals of photosystem I (PS I)[25]. PS I is a $330 \mathrm{kDa}$ membrane protein containing a chain of electron transfer cofactors comprising chlorophyll a, phylloquinones and three $\mathrm{Fe}_{4} \mathrm{~S}_{4}$ clusters that is involved in the light driven electron transport from the lumenal electron donor cytochrome $\mathrm{c}$ or plastocyanin to the stromal electron acceptor ferredoxin in photosynthesis. After collecting detector images from $\sim 1.8$ million X-ray shots, it was possible to identify and index $\sim 15000$ of these as diffraction images from protein crystals. From the indexed diffraction images structure factors were obtained by Monte-Carlo averaging over all the partial observations leading to a structural model at $8.5 \AA$ resolution [25] (Figure 2b). No significant differences were observed with 10 and 70 fs X-ray pulse widths but a decrease in the scattering strength at higher resolution was observed with pulse widths of $200 \mathrm{fs}$. These results indicated that the 10-70 fs pulses are short enough to sample the structure without visible radiation damage, whereas at $200 \mathrm{fs}$ pulse length the irradiation already leads to disordering of the crystal lattice.

This pioneering study of serial femtosecond crystallography (SFX) was soon followed by work on other proteins, including lysozyme [26], cathepsin B [27], and g-protein coupled receptors $[19,28]$. These studies demonstrated that it is possible to achieve close to atomic resolution with SFX [26], that novel structural features can be revealed [27], that structures can be obtained from crystals grown inside living cells [27,29], and that also small membrane protein crystals grown and measured inside lipidic cubic phases can yield high resolution diffraction data 
with this method [28]. In the field of metalloenzymes, the $\mathrm{Zn}$ protease thermolysin [30,31], the soluble electron transfer protein ferredoxin [32], the bacterial reaction center (PBRC) [33], cocrystals of PS I with ferredoxin [34] and micro-crystals of photosystem II (PS II) [31,35-37] (Figure 2) where studied, all using a jet for sample delivery at room temperature. In addition, the goniometer mount method was used for structural studies on the membrane protein complex cytochrome c oxidase $(\mathrm{CcO})$ [11], a [FeFe] hydrogenase [10], myoglobin [10] and PS II [9], using crystals of several $100 \mu \mathrm{m}$ in size frozen at $100 \mathrm{~K}$.

\section{Phasing, anomalous signals from metals/other atoms, and de novo structures}

Diffraction data obtained from crystalline samples using fs XFEL pulses is fundamentally different from standard diffraction measurements at synchrotron sources. The main difference being, that the crystal is not moving during the pulse length, leading to recording of 'stills' in contrast to oscillation images used in SR XRD. In addition by changing the sample volume for recording each individual diffraction image and due to the inherent fluctuations in the intensity and energy spectrum of the XFEL pulses (due to the stochastic nature of the lasing process at an XFEL), there are many potential error sources in the determination of the actual Bragg intensities

for each reflection. Specific new data processing methods have been developed over the last years to handle such data [30,38,39]. Deriving phase information is essential for solving unknown structures but also for obtaining unbiased structural information of a site that might be different from the known homologous structure. Detecting the anomalous signal from metals/S or $\mathrm{Se}$ is one of the methods in XRD to obtain de novo phase information. It has been demonstrated that it is possible to observe small intensity differences between symmetry related Bragg reflections for sulfur in lysozyme [40], for $\mathrm{Zn}, \mathrm{Ca}$ and $\mathrm{S}$ in thermolysin [31] and for Mn in PS II [31] (Figure 2e). In addition, Barends and co-workers demonstrated, using Gd derivatized micro crystals of lysozyme, that it is possible to extract sufficient quality anomalous signal from SFX data to obtain phase information and solve a structure by de-novo phasing [41]. The initial data processing methods often used a 'Monte Carlo' approach of averaging out the many sources 
of error by collecting highly redundant data sets (often 10,000s of diffraction images). More recent developments showed that it is possible to obtain good quality structures by using only a few hundreds of images $[10,42]$ and this field is evolving rapidly.

\section{Establishing structures of undamaged metal sites}

From several initial studies it was found that the "measure before modify" approach in diffraction measurements works for doses in the range of 30-150 MGy using 70 fs and shorter pulses, although indications of possible damage were found for higher doses and longer pulses. In this case the radiation damage is not caused by radical generation but rather by rapid ionization of the sample, followed by coulomb repulsion of the charged ions leading to thermal disorder finally destroying the crystal lattice. The ionization is expected to be element dependent due to different cross section and therefore for example metal sites could be more susceptible to this kind of damage as compared to the low-Z-atoms in the protein.

Recently, a small two $\left[\mathrm{Fe}_{4} \mathrm{~S}_{4}\right]$ cluster containing ferredoxin was used to study the possible effects of radiation damage when exposed to ultra high dose fs X-ray pulses. Nass and coworkers used the $100 \mathrm{~nm}$ focus of the CXI instrument at LCLS to collect datasets above and below the Fe $\mathrm{K}$ edge using a dose of 20-30 GGy [32]. In comparison, the classical damage threshold for protein crystals at synchrotron sources is estimated to be 20-30 MGy under cryo conditions [1,43] and about 0.2 MGy for room temperature experiments [3] and the damage threshold for redox active metal centers was shown to be two to three orders of magnitude less than that required for lattice damage [3].

The electron densities obtained from ferredoxin crystals at LCLS were of sufficient

resolution (2.1-2.5 $\AA$ ) to allow a detailed analysis of the structure of the $\mathrm{Fe}_{4} \mathrm{~S}_{4}$ clusters (Figure 2d). Comparison of the high dose to low dose XFEL and SR data sets showed difference in the Fe positions indicating ionization of the Fe atoms under these conditions. In addition, there was a displacement in the position of the sulfur atoms of the $\mathrm{Fe}_{4} \mathrm{~S}_{4}$ clusters, in agreement with molecular dynamics simulations. Interestingly, the degree of changes in the electron density of 
the $\mathrm{Fe}_{4} \mathrm{~S}_{4}$ clusters was different for the two clusters in the ferredoxin molecule indicating a possible influence of the direct environment and binding site on the disorder caused by the photoionization [32]. It has to be noted that the dose used in this study is about a factor of 1001000 higher than that used for many of the other diffraction experiments discussed in this review. Recently, Chapman et al estimated that with a dose of 400 MGy in an average protein crystal, each atom is ionized once during a FEL pulse. As scattering occurs over the entire pulse length and the earlier parts of the pulse contribute stronger to the signal it was concluded that the scattering signal is predominantly obtained from unmodified (pristine) atoms and that this dose can be used as a threshold to obtain signal before the modification of the electronic structure of the sample [7].

It was possible using XFEL data to obtain the first undamaged structure of the oxidized resting state of cyt c oxidase $(\mathrm{CcO})$, which is a large membrane protein catalyzing the reduction of $\mathrm{O}_{2}$ to water coupled with the generation of a trans-membrane proton gradient. The oxygen reduction takes place at a binuclear active site comprised of copper $\left(\mathrm{Cu}_{\mathrm{B}}\right)$ and a heme iron $\left(\mathrm{Fe}_{\mathrm{a} 3}\right)$. Hirata et al. used the goniometer method to collect single shot still diffraction images from large crystals of bovine cytochrome c oxidase in its fully oxidized resting state frozen at $100 \mathrm{~K}$ [11]. They utilized the $<10$ fs pulses of the XFEL in Japan, SACLA, with a dose of about 9.9 MGy/pulse. By merging intensities obtained from 1400 diffraction images (each one obtained from a fresh sample volume and rotated by $0.1^{\circ}$ with respect to the previous exposure, Fig. 1a) a data set at 1.9 $\AA$ resolution was assembled. By monitoring the intensity of a water peak located close to the active site, which is generated by the reduction of peroxide, Hirata et al. concluded that the XFEL data did not show any radiation damage to the bound peroxide ligand (Figure 1f), whereas synchrotron data sets showed clear X-ray induced peroxide reduction to a hydroxide, forming $\mathrm{Fe}^{3+}-\mathrm{OH}^{-}$and $\mathrm{Cu}^{2+}-\mathrm{OH}^{-}$. In the XFEL data, a peroxide anion with an oxygen-oxygen distance of $1.55 \AA$, similar to O-O distances for typical peroxides, was found and the hydroxide bound state was not present. 
Also using the goniometer method at the XPP endstation of LCLS with <50 fs X-ray pulses and a beam size of 30-50 $\mu \mathrm{m}$, Cohen et al demonstrated the collection of radiation damage free diffraction data from frozen crystals of sperm whale myoglobin at $1.36 \AA$ [10]. In the electron density maps (Figure $1 \mathrm{c}$ ) the heme group was clearly visible, and interestingly, it was possible to obtain a complete dataset out to $1.48 \AA$ resolution using only a subset of 253 out of a total of 739 diffraction images. Using the same setup it was possible to obtain data from a [FeFe]-hydrogenase from C. pasteurianum from five large crystals. In this case 125 still diffraction images, recorded at intervals of $0.5^{\circ}$ rotation, were combined to yield a dataset at 1.6 $\AA$ resolution. The iron sulfur clusters were reliably located in the obtained electron density maps (Figure 1d) with structures nearly identical to previous SR data but with some differences, that are potentially due to radiation damage in the synchrotron data [10].

\section{Following the enzymatic reaction and structural changes in real time under ambient conditions}

In metalloenzymes and proteins in general dynamic processes can be observed over a wide range of time scales (Fig. 3a) and XFELs provide an ideal tool to probe such dynamics. One of the first attempts to follow structural changes in a protein using the fs pulses of an XFEL was the study by Aquila et al on PS I-ferredoxin co-crystals [34]. Upon light excitation of PS I, one electron is transferred from PS I to the natural soluble electron transfer protein ferredoxin, which is bound in its oxidized form but rapidly dissociates from the complex once electron transfer has occurred. Using 0.5-2 $\mu \mathrm{m}$ size crystals, SFX for the dark state as well as $5 \mu$ s, and 10 $\mu$ s after the laser excitation was measured [34] and a slight increase of diffraction intensity at 5 $\mu$ s and a loss at $10 \mu$ s was observed. This was explained by a correlated structural change, followed by disordering of the crystalline lattice that leads to a drop in Bragg intensity in the 10 $\mu$ s data set as the crystals dissolve. 
Studies of fast protein dynamics can also be performed on non-crystalline samples. For example Arnlund and co-workers studied the fast dynamics of the purple bacterial reaction center (PBRC) upon light excitation, measuring wide angle X-ray scattering from protein solutions with different delay times after intense laser excitation using $40 \mathrm{fs}$ X-ray pulses at the CXI instrument of LCLS [44]. At a time resolution of $0.5 \mathrm{ps}$, an ultrafast protein conformational change was observed that peaked at 7 ps and decayed with a half-life of 44 ps (Figure $3 b$ ). The observed change in the solution scattering component attributed to the protein was modeled as a correlated expansion of the trans membrane region of the reaction center by up to $1 \AA$ and it was found that this protein quake away from the central light absorbing co-factors preceded the flow of heat into the protein by approximately 2 ps [44]. A similar approach was used by Levantino et al. [45] to measure structural changes in myoglobin upon light induced photo-dissociation of a CO molecule bound to the heme iron. The time resolved SAXS and WAXS data revealed an ultrafast structural response of the regions of the protein forming the heme binding pocket, oscillating with a period of $\sim 4$ ps [45] (Figure 3c).

When using strongly diffracting crystalline samples, it is also possible to follow subtle changes in side chain positions and co-factor structures upon triggering a reaction. Tenboer et al. recorded time resolved diffraction images from crystals of the photoactive yellow protein (PYP) at delay times of $10 \mathrm{~ns}$ and $1 \mathrm{~ms}$ after laser excitation [46] (Figure 3d-f). From the assembled data sets it was possible to compute differences in the electron density, showing a displacement of the central sulfur atom of the chromophore due to a cis-trans isomerization and corresponding changes in the surrounding amino acids [46].

This approach is also very suitable for following reaction cycles in metalloenzymes, provided a triggering mechanism exists. The light induced reaction cycle at the catalytic center of PS II is a prime example. PS II is a membrane protein complex that catalyzes the light driven oxidation of water to di-oxygen and protons and is integral to all oxygenic photosynthetic organisms. The reaction is catalyzed at a $\mathrm{Mn}_{4} \mathrm{CaO}_{5}$ cluster located at one side of the complex and the metal cluster advances through a cycle of five steps, starting at the dark stable $S_{1}$ state going 
up to the highest oxidized $\mathrm{S}_{3}$ state, that is followed by the actual oxidation of water and release of oxygen and subsequent formation of the lowest oxidized state $\left(\mathrm{S}_{0}\right)$ which in turn after another photon absorption returns to the $S_{1}$ state.

Initial work on femtosecond crystallography of micro crystals of PS II yielded a data set at $6.5 \AA$ resolution of the $\mathrm{S}_{1}$ state at room temperature, showing that the $\mathrm{Mn}_{4} \mathrm{CaO}_{5}$ cluster was present at the expected location despite the dose of $20 \mathrm{MGy} /$ pulse that was used in the study [35]. This study was extended a year later to a higher resolution of 5.7-5.9 $\AA$ for the $S_{1}$ state and the first illuminated $\left(\mathrm{S}_{2}\right)$ state [36]. No obvious differences where visible in the isomorphous difference maps computed between the two datasets, indicating that there are no large scale structural changes occurring upon formation of the $S_{2}$ state. Importantly, by combining the XRD measurements with X-ray spectroscopy it was possible to show that the electronic structure (oxidation state) of the $\mathrm{Mn}_{4} \mathrm{CaO}_{5}$ cluster was not changed due to the fs X-ray pulses (see below) [36].

Using the goniometer method at SACLA, Suga et al succeeded in obtaining a $1.95 \AA$ structure of PS II in the $S_{1}$ state at cryogenic temperature (100 K) [9]. They used crystals of $\sim 1$ $\mathrm{mm}$ in size and collected over 7000 diffraction images from a total of $~ 330$ crystals with a dose of 1.4 MGy/pulse and a pulse duration <10 fs. When comparing the XFEL dataset to previous SR data at similar resolution it became obvious that the Mn-Mn distances within the catalytic cluster are about 0.1-0.2 $\AA$ shorter (Figure 2e), in agreement with earlier X-ray spectroscopy studies [47], most likely due to radiation damage in the SR structures [3,48]. This new undamaged structure gave some insights into the possible valence assignments of the individual Mn due to the Mn-ligand distances and indicated that one of the oxygen ligands (O5), exhibiting longer Mn-O distances, could be a hydroxide and may serve as one of the substrate oxygen atoms in the reaction cycle [9].

In parallel, two groups pursued room temperature studies on microcrystals of PS II in the different intermediate states of the reaction cycle. In the study by Kern et al [31], in-situ illumination of micro crystals was used to populate PS II in the $S_{3}$ and $S_{0}$ state as well as to probe 
a transient state in between the two. To ensure maximum catalytic turnover the illumination conditions were tested in parallel oxygen evolution measurements under similar conditions. Also X-ray emission spectroscopy (XES) data were measured from PS II solutions after 2 flashes, after formation of the $S_{0}$ state (long delay after 3 flashes) and for an intermediate state in between the $S_{3}$ and $S_{0}$ state (250 s after start of the $S_{3}-S_{0}$ transition) (Figure $3 \mathrm{~g}$ ). These data showed a clear shift from oxidized to reduced state between the two conditions ( $2 \mathrm{~F}$ and $3 \mathrm{~F}$ ), corresponding to a majority of the centers in the $S_{3}$ state $(2 F)$ or in the $S_{0}$ state $(3 F)$, respectively. The XES for the intermediate state showed no reduction of $\mathrm{Mn}$, indicating that the $\mathrm{O}-\mathrm{O}$ formation did not start yet, in accordance with previous experiments, demonstrating a lag phase of 150-250 s before the onset of Mn redox chemistry in the $S_{3}-S_{0}$ transition. XRD gave diffraction beyond $4.1 \AA$, and datasets for $2 \mathrm{~F}(\sim 17000$ indexed images, $4.5 \AA$ resolution), 3F ( 13000 images, $4.6 \AA$ resolution), the transient state 3F' ( 7800 images, $5.2 \AA)$ and the dark state (6700 images, $4.9 \AA$ ) were obtained. In addition, an anomalous signal for Mn was observed in all data sets (Figure 1e). In contrast to the results by Kupitz et al [37], no statistically significant changes were observed in the individual $2 \mathrm{Fo}-\mathrm{Fc}$ electron density maps and in the isomorphous difference maps between the different data sets. Based on the signal level it was concluded that changes of an individual Mn atom up to $0.5 \AA$ would not be resolvable but larger shifts would be visible in the data and from this it was concluded that structural changes of $\mathrm{Mn}$ positions in the $\mathrm{Mn}_{4} \mathrm{CaO}_{5}$ cluster between the $S_{1}, S_{3}$ and $S_{0}$ state are smaller than $0.5 \AA$ [31].

The results from the study by Kupitz et al are in contrast to the work by Kern et al. [31]. Kupitz et al measured data in the dark and double illuminated (2F) state [37]. A difference in unit cell volume of $\sim 4 \%$ was observed when comparing the $2 \mathrm{~F}$ with the dark data, and datasets were obtained out to 5.0 and $5.5 \AA$ resolution, respectively. It is noteworthy that there was no independent measure of the turnover of PS II in this study as compared to the study by Kern et al. The comparison of the electron density for the $\mathrm{Mn}_{4} \mathrm{CaO}_{5}$ cluster to the $\mathrm{SR}$ high resolution data [49] led to an interpretation that a more compact form was observed in the XFEL study. From the comparison between the electron density for both datasets Kupitz et al also concluded that 
there are differences in loop regions around the $\mathrm{Mn}_{4} \mathrm{CaO}_{5}$ cluster and the electron acceptor side, next to the non-heme iron and the two quinone cofactors. The observed changes in the electron density were interpreted as suggestive of an increase in the distance between the $\mathrm{Mn}_{3}$ cubane like unit of the cluster and the more isolated $\mathrm{Mn}$ and a distortion in the cubane in the 2F data [37].

\section{Room temperature, real-time X-ray spectroscopy}

The inherent element specificity of X-ray spectroscopy is ideally suited to follow changes at specific metal sites against a large background of low $\mathrm{Z}$ atoms, as is the case in a metalloenzyme. X-ray absorption spectroscopy of transition metals at the K-edge follows changes in the lowest unoccupied orbitals by probing the $1 \mathrm{~s}-3 \mathrm{p} / \mathrm{d}$ transition. Complementary to this, $\mathrm{K} \beta$ emission spectroscopy probes the highest occupied orbitals by measuring the fluorescence emitted upon decay of a $3 \mathrm{p}$ electron back into a 1 s core hole created by the initial X-ray probe (see Figure 4a). Due to the strong exchange interaction between the metal 3p and $3 \mathrm{~d}$ orbitals, the emission lines are sensitive to the chemical nature of the metal, e.g. its spin and oxidation state [50]. A more direct probe of the $3 \mathrm{~d}$ orbitals is possible in transition metal L-edge spectroscopy, where the $2 \mathrm{p}$ to $3 \mathrm{~d}$ transition is directly probed (Figure $4 \mathrm{a}$ ). Both methods are currently being used at XFEL sources and will be summarized here. XES has the advantage of being independent of the excitation energy, as long as the energy is above the absorption edge of the metal. Therefore no monochromatization is necessary and the full SASE bandwidth of the XFEL pulse can be used. K $\beta$ XES on dilute solution samples was initially demonstrated for Mn compounds [51] showing that the fs pulses did not perturb the electronic structure of the compounds, including higher valent $\mathrm{Mn}$ complexes that were studied $\left(\mathrm{Mn}^{\mathrm{II}}\right.$ and $\mathrm{Mn}^{\mathrm{IIIIV}}$ ) (Figure 4c). For iron(II) tris(2,2' -bipyridine), it was also possible to record time resolved XES at 50 fs to 1 ps after laser excitation and resolve the kinetics of the light induced spin change [52,53] using $50 \mathrm{mM}$ concentration in a $100 \mathrm{~mm}$ thick liquid jet. The same system was also studied by transient XAS with similar time resolution [54]. Despite the need to use a monochromator, 
resulting in a lower X-ray intensity on the sample and longer acquisition times, good quality transient spectra were recorded (Figure $4 b$ ).

XES is well suited to be used simultaneously with other techniques, because of the flexibility in the requirements on the X-ray probe energy. One experimental setup that has been successfully used for combining XES and XRD is shown in Figure 1b. A study on microcrystals of PS II was performed (see above), measuring both the diffraction and the emission signal simultaneously, from the same sample volume. This combined approach makes it possible to determine that the electronic structure of the highly radiation sensitive $\mathrm{Mn}_{4} \mathrm{CaO}_{5}$ cluster in PS II is not altered by the probe conditions used at LCLS; an important prerequisite for the detailed interpretation of the structural data. In addition, the spectroscopic information provides confirmation of the catalytic turnover under the experimental conditions and the spectra independently provide insights into the evolution of the electronic structure over the reaction cycle. In the recent work by Kern et al., XES was used to track the changes in oxidation state of $\mathrm{Mn}_{4} \mathrm{CaO}_{5}$ cluster in PS II over time during the $\mathrm{S}_{3}-\mathrm{S}_{0}$ transition [31]. The transient data for a time point $250 \mathrm{~s}$ after the laser pump (Figure $3 \mathrm{f}$ ) was used to conclude that no $\mathrm{Mn}$ redox chemistry had occurred by that time interval, suggesting that the O-O bond formation had not yet occurred within the interval of $250 \mu \mathrm{s}$ after the third visible laser flash illumination. One can also envision extending this approach to multiple element systems, by following the emission signal of two elements concurrently. A demonstration experiment of such a system was performed recently at the ALS using a mixed MnNi oxide [55].

Transition metal L-edge spectroscopy is an ideal method to obtain more detailed information on the electronic structure of a metal center.. In the L-edge region the spectral features are more highly resolved due to the narrower line width and the d-orbitals are directly probed (2p-3d transition, Figure $4 a)$. However, due to the large absorption cross sections at soft X-ray energies, the damage threshold is much lower than at hard X-ray energies and it is practically impossible to collect undamaged L-edge spectra from metalloenzymes or metal complexes in aqueous solution under ambient condition at SR sources. Recently, a first 
demonstration experiment at LCLS was conducted showing the feasibility of collecting Mn Ledge spectra from aqueous solutions at room temperature using a reflection zone plate spectrometer [56] (Figure 4d). One step further would be the collection of L-edge resonant inelastic X-ray scattering data (RIXS), where one can map the energy levels and occupation of the valence orbitals. At present only K-edge RIXS studies have been conducted on metalloenzymes [57,58], but a first demonstration experiment at LCLS showed that it is possible to collect time resolved L-edge RIXS on a solution of an Fe compound in in the $\sim 100 \mathrm{~s} \mathrm{mM}$ concentration range [59].

\section{Conclusions, Potential and Outlook}

The ultra fast high intensity X-ray pulses of XFEL sources open up completely new possibilities for the study of metalloenzymes. There are two major advantages. First, the fs pulses make it possible to monitor processes that occur in the ps and sub-ps time scale, studies that at normal synchrotron sources are limited to about 100 ps time resolution. In addition, the ultra short pulses allow one to outrun X-ray induced modification of the sample, thereby enabling the study of un-perturbed states while applying a very high X-ray dose to the sample and performing such experiments at room temperature and in an aqueous environment. Therefore it becomes possible to follow changes in the electronic and geometric structure of metal containing enzymes, in real time, using diffraction and X-ray spectroscopy methods. The first snap shots of such reaction movies have already been obtained, and it is expected that with the opening of additional XFEL facilities in the next few years this field will greatly expand and provide novel insights into the important reaction mechanisms of metalloenzymes, that are at the heart of many essential reactions in biology.

\section{Acknowledgements}

The authors acknowledge research support from the Director, Office of Science, Office of Basic Energy Sciences, Division of Chemical Sciences, Geosciences, and Biosciences of the 
Department of Energy (DOE) under Contract DE-AC02-05CH11231 (J.Y and V.K.Y), the NIH Grants GM55302 (V.K.Y) and GM110501 (J.Y.), the Alexander von Humboldt Foundation (J.K.), and the Human Frontier Research grant RGP0063/2013 (J.Y.). We are grateful to our present and former group members and all our collaborators, who contributed to the research from our group presented in this review. 


\section{References}

1. Garman EF, Weik M: Radiation damage to macromolecules: kill or cure? J Synchrotron Radiat 2015, 22:195-200.

2. Sigfridsson KG, Chernev P, Leidel N, Popovic-Bijelic A, Graslund A, Haumann M: Rapid Xray photoreduction of dimetal-oxygen cofactors in ribonucleotide reductase. $\mathrm{J}$ Biol Chem 2013, 288:9648-9661.

3. Yano J, Kern J, Irrgang KD, Latimer MJ, Bergmann U, Glatzel P, Pushkar Y, Biesiadka J, Loll B, Sauer K, et al.: X-ray damage to the $\mathrm{Mn}_{4} \mathrm{Ca}$ complex in single crystals of photosystem II: a case study for metalloprotein crystallography. Proc Natl Acad Sci U S A 2005, 102:12047-12052.

4. Emma P, Akre R, Arthur J, Bionta R, Bostedt C, Bozek J, Brachmann A, Bucksbaum P, Coffee R, Decker FJ, et al.: First lasing and operation of an ångstrom-wavelength freeelectron laser. Nature Photonics 2010, 4:641-647.

5. Ishikawa T, Aoyagi H, Asaka T, Asano Y, Azumi N, Bizen T, Ego H, Fukami K, Fukui T, Furukawa Y, et al.: A compact X-ray free-electron laser emitting in the sub-ångström region. Nature Photonics 2012, 6:540-544.

6. Neutze R, Wouts R, van der Spoel D, Weckert E, Hajdu J: Potential for biomolecular imaging with femtosecond X-ray pulses. Nature 2000, 406:752-757. 
7. Chapman HN, Caleman C, Timneanu N: Diffraction before destruction. Philos Trans R Soc Lond B Biol Sci 2014, 369:20130313.

8. Margaritondo G, Rebernik Ribic P: A simplified description of X-ray free-electron lasers. $J$ Synchrotron Radiat 2011, 18:101-108.

9. Suga M, Akita F, Hirata K, Ueno G, Murakami H, Nakajima Y, Shimizu T, Yamashita K, Yamamoto $\mathrm{M}$, Ago $\mathrm{H}$, et al.: Native structure of photosystem II at $1.95 \AA$ resolution viewed by femtosecond X-ray pulses. Nature 2015, 517:99-103.

**Damage free high resolution structure of photosytem II, recorded at $100 \mathrm{~K}$, with metal-metal distances in agreement with X-ray spectroscopy. The structure gave insight about important details of the $\mathrm{Mn}_{4} \mathrm{CaO}_{5}$ cluster, regarding possible valence assignment.

10. Cohen AE, Soltis SM, Gonzalez A, Aguila L, Alonso-Mori R, Barnes CO, Baxter EL, Brehmer W, Brewster AS, Brunger AT, et al.: Goniometer-based femtosecond crystallography with X-ray free electron lasers. Proc Natl Acad Sci U A 2014, 111:17122-17127.

* First results from gonimoeter based cryo crystallography at LCLS, reporting structures from myoglobin and FeFe hydrogenase at high resolution. It was possible to obtain good quality data sets with only a few hundred diffraction images.

11. Hirata K, Shinzawa-Itoh K, Yano N, Takemura S, Kato K, Hatanaka M, Muramoto K, Kawahara T, Tsukihara T, Yamashita E, et al.: Determination of damage-free crystal structure of an X-ray-sensitive protein using an XFEL. Nat Methods 2014, 11:734-736.

** High resolution structure of cyt c oxidase using large crystals at $100 \mathrm{~K}$ and the goniometer method. The structure of the active site shows the presence of a peroxide between the heme Fe and the $\mathrm{Cu} \mathrm{B}$ center, whereas synchrotron data only revealed the presence of a hydroxide form, due to radiation damage. 
12. Hunter MS, Segelke B, Messerschmidt M, Williams GJ, Zatsepin NA, Barty A, Benner WH, Carlson DB, Coleman M, Graf A, et al.: Fixed-target protein serial microcrystallography with an x-ray free electron laser. Sci Rep 2014, 4:6026.

13. Feld GK, Frank M: Enabling membrane protein structure and dynamics with X-ray free electron lasers. Curr Opin Struct Biol 2014, 27:69-78.

14. Frank M, Carlson DB, Hunter MS, Williams GJ, Messerschmidt M, Zatsepin NA, Barty A, Benner WH, Chu K, Graf AT, et al.: Femtosecond X-ray diffraction from twodimensional protein crystals. IUCrJ 2014, 1:95-100.

15. Shapiro DA, Chapman HN, Deponte D, Doak RB, Fromme P, Hembree G, Hunter M, Marchesini S, Schmidt K, Spence J, et al.: Powder diffraction from a continuous microjet of submicrometer protein crystals. J Synchrotron Radiat 2008, 15:593-599.

16. Weierstall U: Liquid sample delivery techniques for serial femtosecond crystallography. Philos Trans R Soc Lond B Biol Sci 2014, 369:20130337.

17. Sierra RG, Laksmono H, Kern J, Tran R, Hattne J, Alonso-Mori R, Lassalle-Kaiser B, Glockner C, Hellmich J, Schafer DW, et al.: Nanoflow electrospinning serial femtosecond crystallography. Acta Crystallogr D Biol Crystallogr 2012, 68:1584-1587.

18. Kern J, Hattne J, Tran R, Alonso-Mori R, Laksmono H, Gul S, Sierra RG, Rehanek J, Erko A, Mitzner R, et al.: Methods development for diffraction and spectroscopy studies of metalloenzymes at X-ray free-electron lasers. Philos Trans R Soc Lond B Biol Sci 2014, 369:20130590. 
19. Liu W, Wacker D, Wang C, Abola E, Cherezov V: Femtosecond crystallography of membrane proteins in the lipidic cubic phase. Philos Trans R Soc Lond B Biol Sci 2014, 369:20130314.

20. Weierstall U, James D, Wang C, White TA, Wang D, Liu W, Spence JC, Bruce Doak R, Nelson G, Fromme P, et al.: Lipidic cubic phase injector facilitates membrane protein serial femtosecond crystallography. Nat Commun 2014, 5:3309.

21. Ekeberg T, Svenda M, Abergel C, Maia FRNC, Seltzer V, Claverie J-M, Hantke M, Jönsson O, Nettelblad C, van der Schot G, et al.: Three-dimensional reconstruction of the giant mimivirus particle with an X-Ray Free-Electron Laser. Physical Review Letters 2015, 114.

22. Hantke MF, Hasse D, Maia FRNC, Ekeberg T, John K, Svenda M, Loh ND, Martin AV, Timneanu N, Larsson DSD, et al.: High-throughput imaging of heterogeneous cell organelles with an X-ray laser. Nature Photonics 2014, 8:943-949.

23. Seibert MM, Ekeberg T, Maia FR, Svenda M, Andreasson J, Jonsson O, Odic D, Iwan B, Rocker A, Westphal D, et al.: Single mimivirus particles intercepted and imaged with an X-ray laser. Nature 2011, 470:78-81.

24. Bogan MJ: X-ray free electron lasers motivate bioanalytical characterization of protein nanocrystals: serial femtosecond crystallography. Anal Chem 2013, 85:3464-3471.

25. Chapman HN, Fromme P, Barty A, White TA, Kirian RA, Aquila A, Hunter MS, Schulz J, DePonte DP, Weierstall U, et al.: Femtosecond X-ray protein nanocrystallography. Nature 2011, 470:73-77. 
26. Boutet S, Lomb L, Williams GJ, Barends TR, Aquila A, Doak RB, Weierstall U, DePonte DP, Steinbrener J, Shoeman RL, et al.: High-resolution protein structure determination by serial femtosecond crystallography. Science 2012, 337:362-364.

27. Redecke L, Nass K, DePonte DP, White TA, Rehders D, Barty A, Stellato F, Liang M, Barends TR, Boutet S, et al.: Natively inhibited Trypanosoma brucei cathepsin B structure determined by using an X-ray laser. Science 2013, 339:227-230.

28. Liu W, Wacker D, Gati C, Han GW, James D, Wang D, Nelson G, Weierstall U, Katritch V, Barty A, et al.: Serial femtosecond crystallography of $\mathbf{G}$ protein-coupled receptors. Science 2013, 342:1521-1524.

29. Sawaya MR, Cascio D, Gingery M, Rodriguez J, Goldschmidt L, Colletier JP, Messerschmidt MM, Boutet S, Koglin JE, Williams GJ, et al.: Protein crystal structure obtained at 2.9 $\AA$ resolution from injecting bacterial cells into an X-ray free-electron laser beam. Proc Natl Acad Sci U S A 2014, 111:12769-12774.

30. Hattne J, Echols N, Tran R, Kern J, Gildea RJ, Brewster AS, Alonso-Mori R, Glockner C, Hellmich J, Laksmono $\mathrm{H}$, et al.: Accurate macromolecular structures using minimal measurements from X-ray free-electron lasers. Nat Methods 2014, 11:545-548.

* Analysis of XFEL crystallography data that requires fewer images than previously thought necessary with demonstrations using thermolysin XFEL data.

31. Kern J, Tran R, Alonso-Mori R, Koroidov S, Echols N, Hattne J, Ibrahim M, Gul S, Laksmono H, Sierra RG, et al.: Taking snapshots of photosynthetic water oxidation using femtosecond X-ray diffraction and spectroscopy. Nat Commun 2014, 5:4371. 
* Combined X-ray diffraction and spectroscopy study on micro crystals of PS II at room temperature of different intermediate states generated by in situ illumination with a visible laser. The data at 4.5-5 ̊ resolution revealed no large scale structural changes in the catalytic site, and the emission spectra showed that no oxidation state change occured in the first 250 $\mu$ s during the slow $S_{3}-S_{0}$ transition.

32. Nass K, Foucar L, Barends TR, Hartmann E, Botha S, Shoeman RL, Doak RB, Alonso-Mori $\mathrm{R}$, Aquila $\mathrm{A}$, Bajt $\mathrm{S}$, et al.: Indications of radiation damage in ferredoxin microcrystals using high-intensity X-FEL beams. J Synchrotron Radiat 2015, 22:225-238.

33. Johansson LC, Arnlund D, Katona G, White TA, Barty A, DePonte DP, Shoeman RL, Wickstrand C, Sharma A, Williams GJ, et al.: Structure of a photosynthetic reaction centre determined by serial femtosecond crystallography. Nat Commun 2013, 4:2911.

34. Aquila A, Hunter MS, Doak RB, Kirian RA, Fromme P, White TA, Andreasson J, Arnlund D, Bajt S, Barends TR, et al.: Time-resolved protein nanocrystallography using an X-ray free-electron laser. Opt Express 2012, 20:2706-2716.

35. Kern J, Alonso-Mori R, Hellmich J, Tran R, Hattne J, Laksmono H, Glockner C, Echols N, Sierra RG, Sellberg J, et al.: Room temperature femtosecond X-ray diffraction of photosystem II microcrystals. Proc Natl Acad Sci U S A 2012, 109:9721-9726.

36. Kern J, Alonso-Mori R, Tran R, Hattne J, Gildea RJ, Echols N, Glockner C, Hellmich J, Laksmono H, Sierra RG, et al.: Simultaneous femtosecond X-ray spectroscopy and diffraction of photosystem II at room temperature. Science 2013, 340:491-495.

** First simultaneous crystallography and spectroscopy measurements at an XFEL, showing that intact structures at room temperature can be obtained and that electronic structure of the Mn cluster is not altered by the intense fs X-ray pulses. 
37. Kupitz C, Basu S, Grotjohann I, Fromme R, Zatsepin NA, Rendek KN, Hunter MS, Shoeman RL, White TA, Wang D, et al.: Serial time-resolved crystallography of photosystem II using a femtosecond X-ray laser. Nature 2014, 513:261-265.

38. White TA, Barty A, Stellato F, Holton JM, Kirian RA, Zatsepin NA, Chapman HN: Crystallographic data processing for free-electron laser sources. Acta Crystallogr D Biol Crystallogr 2013, 69:1231-1240.

39. Barty A, Kirian RA, Maia FR, Hantke M, Yoon CH, White TA, Chapman H: Cheetah: software for high-throughput reduction and analysis of serial femtosecond X-ray diffraction data. J Appl Crystallogr 2014, 47:1118-1131.

40. Barends TR, Foucar L, Shoeman RL, Bari S, Epp SW, Hartmann R, Hauser G, Huth M, Kieser C, Lomb L, et al.: Anomalous signal from $\mathbf{S}$ atoms in protein crystallographic data from an X-ray free-electron laser. Acta Crystallogr D Biol Crystallogr 2013, 69:838842.

41. Barends TR, Foucar L, Botha S, Doak RB, Shoeman RL, Nass K, Koglin JE, Williams GJ, Boutet S, Messerschmidt M, et al.: De novo protein crystal structure determination from X-ray free-electron laser data. Nature 2014, 505:244-247.

42. Uervirojnangkoorn M, Zeldin OB, Lyubimov AY, Hattne J, Brewster AS, Sauter NK, Brunger AT, Weis WI: Enabling X-ray free electron laser crystallography for challenging biological systems from a limited number of crystals. Elife 2015, 4. 
43. Henderson R: Cryoprotection of protein crystals against radiation damage in electron and x-ray diffraction. Proceedings of the Royal Society of London, Series B: Biological Sciences 1990, 241:6-8.

44. Arnlund D, Johansson LC, Wickstrand C, Barty A, Williams GJ, Malmerberg E, Davidsson J, Milathianaki D, DePonte DP, Shoeman RL, et al.: Visualizing a protein quake with time-resolved X-ray scattering at a free-electron laser. Nat Methods 2014, 11:923-926.

* fs solution scattering study showing response of the protein framework upon light excitation of the reaction center within a few ps.

45. Levantino M, Schiro G, Lemke HT, Cottone G, Glownia JM, Zhu D, Chollet M, Ihee H, Cupane A, Cammarata M: Ultrafast myoglobin structural dynamics observed with an Xray free-electron laser. Nat Commun 2015, 6:6772.

* fs solution scattering study showing response of the protein framework upon CO dissociation in myoglobin within a few ps.

46. Tenboer J, Basu S, Zatsepin N, Pande K, Milathianaki D, Frank M, Hunter M, Boutet S, Williams GJ, Koglin JE, et al.: Time-resolved serial crystallography captures highresolution intermediates of photoactive yellow protein. Science 2014, 346:1242-1246.

47. Yano $\mathrm{J}$, Yachandra V: $\mathbf{M n}_{4}$ Ca cluster in photosynthesis: where and how water is oxidized to dioxygen. Chem Rev 2014, 114:4175-4205.

48. Glockner C, Kern J, Broser M, Zouni A, Yachandra V, Yano J: Structural changes of the oxygen-evolving complex in photosystem II during the catalytic cycle. J Biol Chem 2013, 288:22607-22620.

49. Umena Y, Kawakami K, Shen JR, Kamiya N: Crystal structure of oxygen-evolving photosystem II at a resolution of 1.9 ̊. Nature 2011, 473:55-60. 
50. Glatzel P, Bergmann U: High resolution 1s core hole X-ray spectroscopy in 3d transition metal complexes-electronic and structural information. Coordination Chemistry Reviews 2005, 249:65-95.

51. Alonso-Mori R, Kern J, Gildea RJ, Sokaras D, Weng TC, Lassalle-Kaiser B, Tran R, Hattne J, Laksmono H, Hellmich J, et al.: Energy-dispersive X-ray emission spectroscopy using an X-ray free-electron laser in a shot-by-shot mode. Proc Natl Acad Sci U S A 2012, 109:19103-19107.

52. Zhang W, Alonso-Mori R, Bergmann U, Bressler C, Chollet M, Galler A, Gawelda W, Hadt RG, Hartsock RW, Kroll T, et al.: Tracking excited-state charge and spin dynamics in iron coordination complexes. Nature 2014, 509:345-348.

53. Bertoni R, Cammarata M, Lorenc M, Matar SF, Letard JF, Lemke HT, Collet E: Ultrafast light-induced spin-state trapping photophysics investigated in $\mathrm{Fe}(\mathrm{phen})_{2}(\mathrm{NCS})_{2}$ spincrossover crystal. Acc Chem Res 2015, 48:774-781.

54. Lemke HT, Bressler C, Chen LX, Fritz DM, Gaffney KJ, Galler A, Gawelda W, Haldrup K, Hartsock RW, Ihee H, et al.: Femtosecond X-ray absorption spectroscopy at a hard X-ray free electron laser: application to spin crossover dynamics. J Phys Chem A 2013, 117:735-740.

55. Gul S, Ng JW, Alonso-Mori R, Kern J, Sokaras D, Anzenberg E, Lassalle-Kaiser B, Gorlin Y, Weng TC, Zwart PH, et al.: Simultaneous detection of electronic structure changes from two elements of a bifunctional catalyst using wavelength-dispersive X-ray emission spectroscopy and in situ electrochemistry. Phys Chem Chem Phys 2015. 
56. Mitzner R, Rehanek J, Kern J, Gul S, Hattne J, Taguchi T, Alonso-Mori R, Tran R, Weniger C, Schroder H, et al.: L-Edge X-ray absorption spectroscopy of dilute systems relevant to metalloproteins using an X-ray Free-Electron Laser. J Phys Chem Lett 2013, 4:36413647.

* Demonstration of soft X-ray L-edge spectroscopy of dilute samples of transition metals at room temperature at an XFEL.

57. Kroll T, Hadt RG, Wilson SA, Lundberg M, Yan JJ, Weng TC, Sokaras D, Alonso-Mori R, Casa D, Upton MH, et al.: Resonant inelastic X-ray scattering on ferrous and ferric bisimidazole porphyrin and cytochrome c: nature and role of the axial methionine-Fe bond. J Am Chem Soc 2014, 136:18087-18099.

58. Glatzel P, Schroeder H, Pushkar Y, Boron T, 3rd, Mukherjee S, Christou G, Pecoraro VL, Messinger J, Yachandra VK, Bergmann U, et al.: Electronic structural changes of Mn in the oxygen-evolving complex of photosystem II during the catalytic cycle. Inorg Chem 2013, 52:5642-5644.

59. Wernet P, Kunnus K, Josefsson I, Rajkovic I, Quevedo W, Beye M, Schreck S, Gru bel S, Scholz M, Nordlund D, et al.: Orbital-specific mapping of the ligand exchange dynamics of $\mathbf{F e}(\mathbf{C O})_{5}$ in solution. Nature 2015, 520:78-81. 


\section{Figure Legends:}

Figure 1: Goniometer based crystallography experiments of metalloenzymes at XFEL sources. (a) Schematic of the experimental approach using a large crystal to collect still shots in a defined orientation with respect to each other, translating by $50 \mathrm{~mm}$ between shots and rotating by a small angle [11]. (b) Alternatively many small crystals mounted in a mesh or similarly can be used. The few mm focused beam leaves imprints on the sample [10]. (c) Omit electron density for the heme group in myoglobin obtained from measuring diffraction of several hundred individual small crystals [10]. (d) 2Fo - Fc (blue, contoured at $1.0 \sigma$ ) and Fo - Fc (red, contoured at $3.0 \sigma$ ) difference density maps calculated for [FeFe] hydrogenase at the site of one of the Fe-S clusters obtained from measurements on five large crystals [10]. (e) Positions of the atoms in the catalytic center of PS II as obtained from cryo SFX is shown in comparison to the positions obtained by SR experiments (blue spheres) [9]. (f) Radiation damage free omit map for the $\mathrm{Fe} \mathrm{Cu}$ center of $\mathrm{CoC}$ showing the position of the peroxide ligand [11].

Figure 2: Jet based crystallography experiments of metalloenzymes at XFEL sources. (a) Examples of nano- to micrometer size crystals used, e.g. thermolysin [17], PS II [36] and toxin crystals (grown inside cells) [29]. (b) Schematic of the experimental approach using a liquid jet for sample delivery to the interaction point and collecting the forward scattering is shown. This setup can be combined with triggering by optical laser pulses and with spectroscopic measurements, e.g. X-ray emission spectroscopy. XFEL structures obtained: (c) PS I with electron density shown in blue and omit maps for the $\mathrm{Fe}_{4} \mathrm{~S}_{4}$ clusters in red [25]. (d) Electron density for one of the $\mathrm{Fe}_{4} \mathrm{~S}_{4}$ clusters in ferredoxin, with omit map shown in green [32]. (e) PS II 
with overall structure of one monomer (bottom left), the oxygen evolving complex (bottom right) [36] and the anomalous difference density from the Mn atoms in the OEC (top right) [31]. (f) PBRC with overall structure and omit maps for the cofactors (left) and detail for the electron density obtained for one of the heme groups (right) [33].

Figure 3: Time resolved experiments using fs X-ray pulses at the CXI instrument at LCLS. (a) Timescales for dynamic processes in proteins/enzymes. (b) Time resolved WAXS measurements on the photosynthetic bacterial reaction center. Upon light excitation a ultrafast protein movements in the ps time regime was visible [44]. (c) Illustration of the protein-quake observed within a few ps in myoglobin upon photodissociation of CO [45], red shaded areas are involved in the movement leading to a volume increase of $\sim 200 \AA^{3}$. (d-f) Time resolved serial femtosecond crystallography on photoactive yellow protein, showing the electron density for (d) the dark state as well as difference electron density for (e) data recorded $10 \mathrm{~ns}$ after light excitation and (f) $1 \mathrm{~ms}$ after light excitation. A movement of the S-atom of the chromophore is visible already in the $10 \mathrm{~ns}$ data (Adapted from [46]). (g) X-ray emission spectra of $\mathrm{Mn}$ in photosystem II. Spectra are shown for the $2 \mathrm{~F}$ state $\left(\mathrm{S}_{3}\right)$, as well as for a intermediate $250 \mathrm{~ms}$ after the $3^{\text {rd }}$ light flash and $0.5 \mathrm{~s}$ after the $3^{\text {rd }}$ light flash $\left(\mathrm{S}_{0}\right.$-state). The difference spectra (middle panel) indicate a reduction in the $\mathrm{S}_{3}-\mathrm{S}_{0}$ transition (as expected from SR measurements - bottom panel), but no specific reduction is visible for the $250 \mathrm{~ms}$ state [31].

Figure 4: X-ray spectroscopic measurements using XFEL pulses. (a) Energy level diagram illustrating the transitions involved in L- and K-edge transition metal spectroscopy. (b) Examples for Fe K-edge absorption spectra showing transient changes in spin state [53]. (c) Experimental setup for collecting $\mathrm{K} \beta$ emission spectra using a dispersive Von Hamos spectrometer and a sample injector. In addition spectra of two Mn compounds collected at LCLS are shown [51]. (d) Experimental setup for collection of Mn L-edge spectra at an XFEL from dilute aqueous 
solutions of Mn compounds using a reflective zone plate (RZP) spectrometer. The spectrum shown was recorded at LCLS using $500 \mathrm{mM} \mathrm{MnCl}_{2}$ in water [56]. 
(a)

(b)
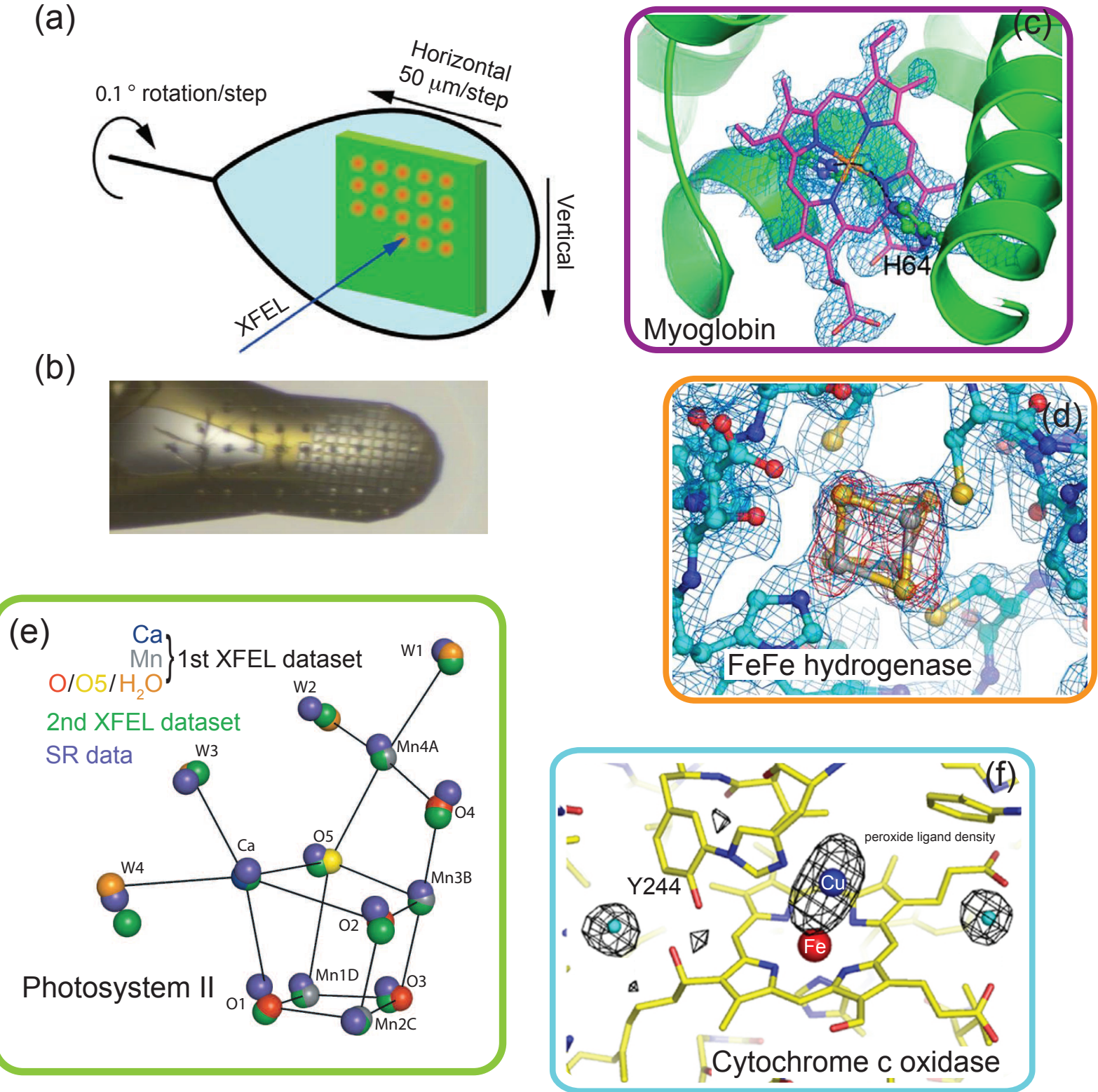
Figure 2

(a)

Nano/Microcrystals

Cry3A toxin crystals inside Bacillus thuringiensis cells
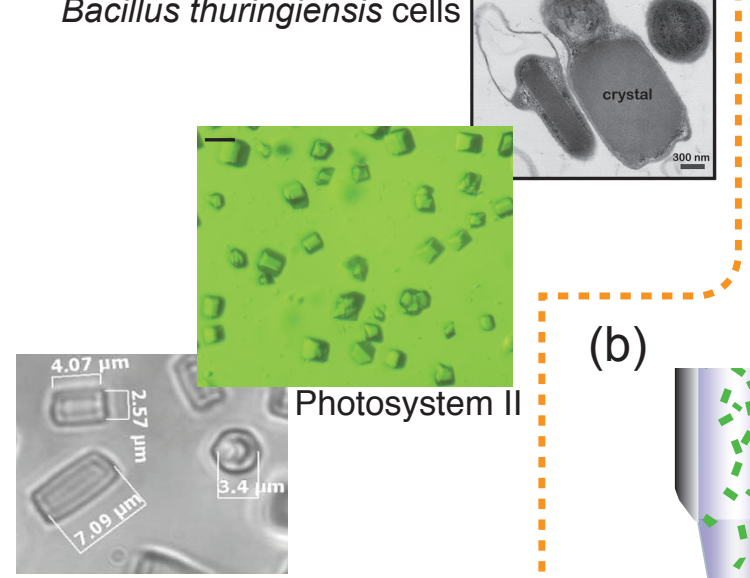

(b)

Thermolysin

(f)
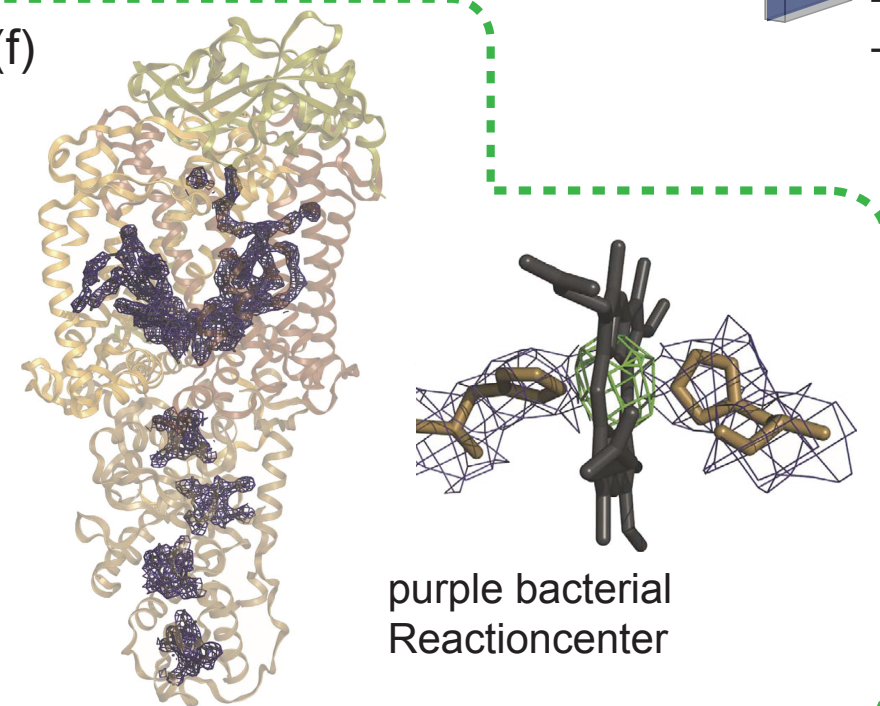

X-ray spectroscopy

fs X-ray pulses

- emission

- absorption

purple bacterial

Reactioncenter

Photosystem II

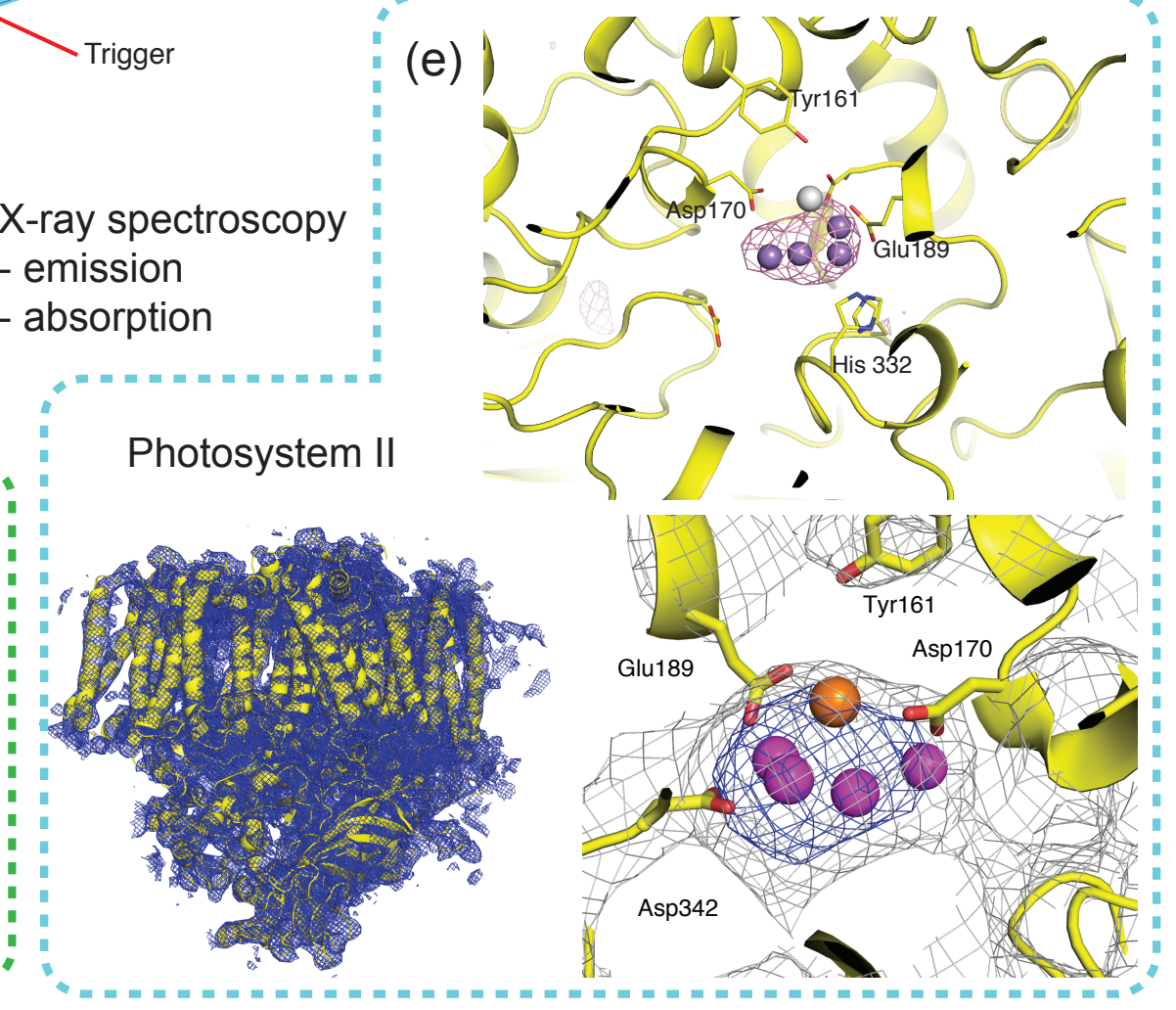

(d) Ferredoxin

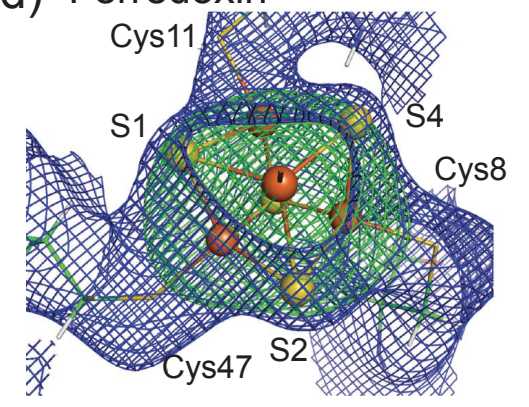

X-ray crystallography $X$-ray solution scattering

Photosystem I 
Figure 3

(a)

$\begin{array}{cccccc}\text { fs } & \text { ps } & \text { ns } & \mu s & \text { ms } & \text { s } \\ 10^{-15} & 10^{-13} & 10^{-9} & 10^{-6} & 10^{-3} & 10^{0} \mathrm{sec}\end{array}$

exciton transfer

proton transfer

vibrational motions

(b)

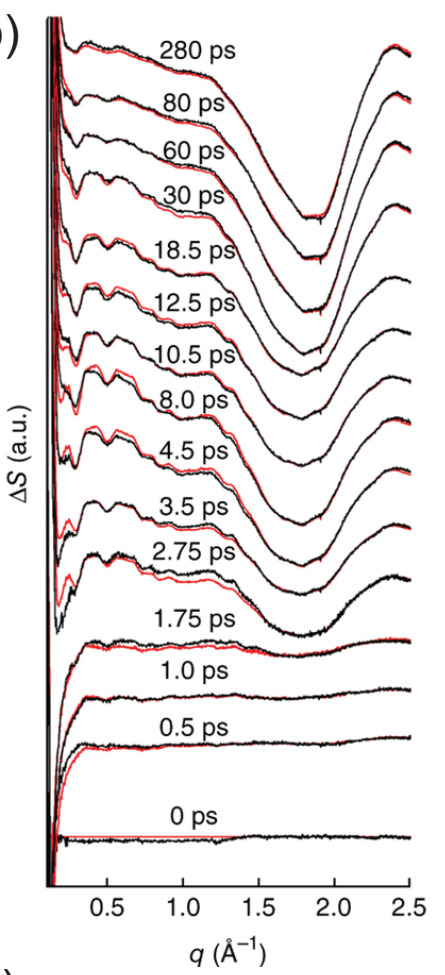

(c)

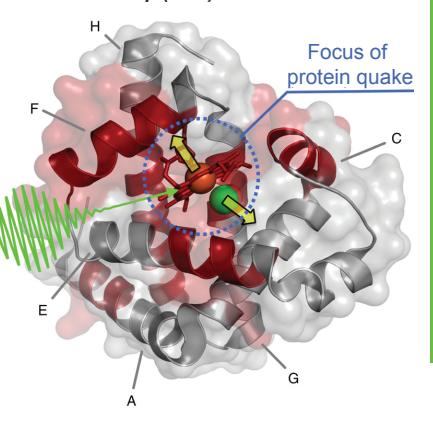

electron transfer

intra - inter-molecular protein conformational changes enzyme turnover
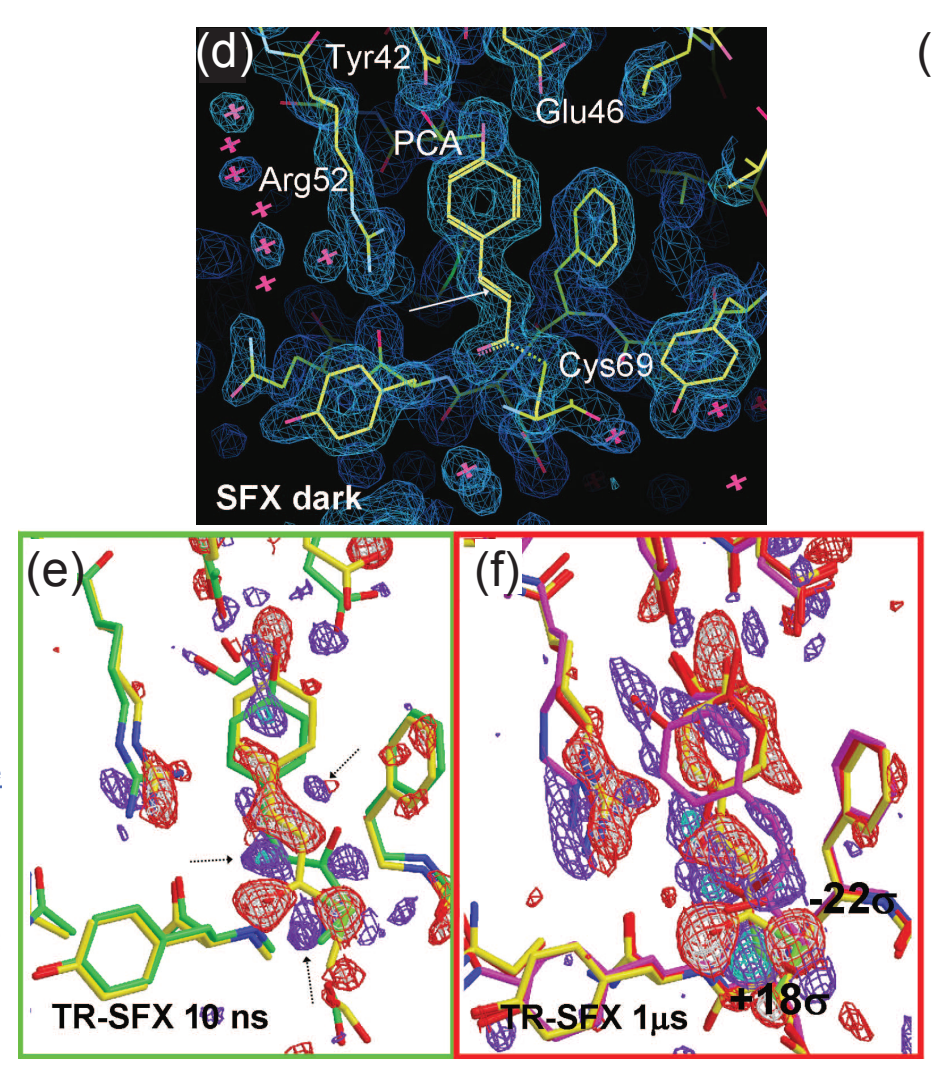

(g)

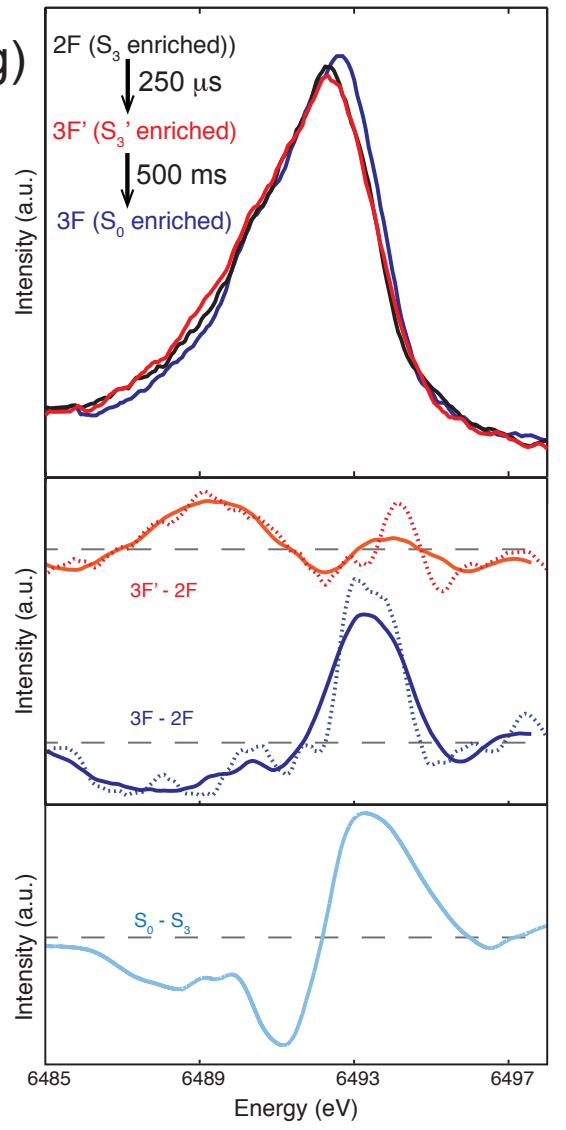




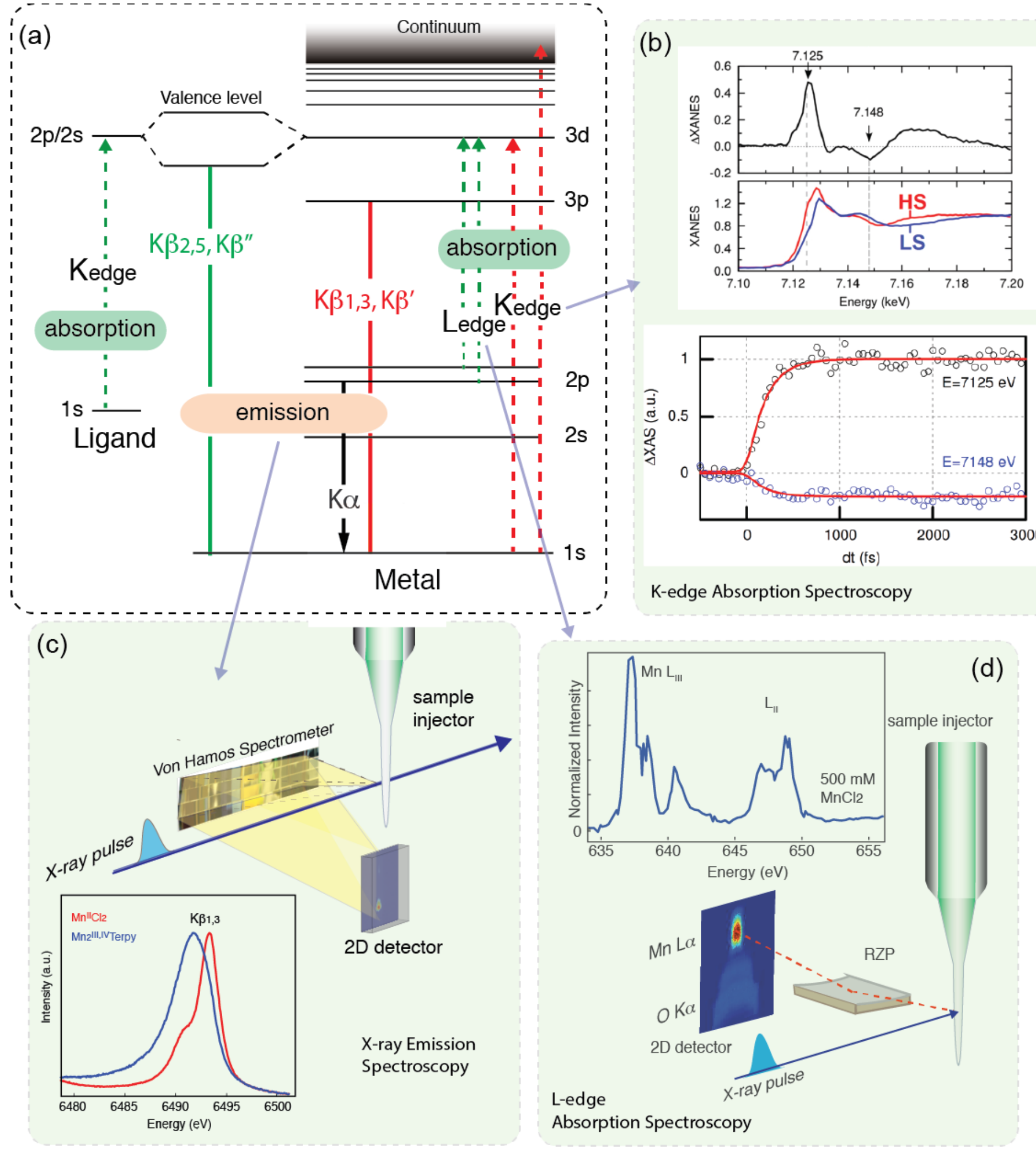

